\title{
The Hawking Effect for Massive Particles
}

\author{
Bernard R. Durney \\ Route de Carcés, Lorgues, France \\ Email: durney@physics.arizona.edu
}

Received 20 November 2013; revised 15 December 2013; accepted 24 December 2013

Copyright (C) 2014 by author and Scientific Research Publishing Inc. This work is licensed under the Creative Commons Attribution International License (CC BY). http://creativecommons.org/licenses/by/4.0/

\section{(c) (i) Open Access}

\section{Abstract}

This paper describes a particularly transparent derivation of the Hawking effect for massive particles in black holes. The calculations are performed with the help of Painlevé-Gullstrand's coordinates which are associated with a radially free-falling observer that starts at rest from infinity. It is shown that if the energy per unit rest mass, $e$, is assumed to be related to the Killing constant, $k$, by $k^{2}=2 e-1$ then $e$, must be greater than $\frac{1}{2}$. For particles that are confined below the event horizon (EH), $k$ is negative. In the quantum creation of particle pairs at the EH with $k=1$, the time component of the particle's four velocity that lies below the EH is compatible only with the time component of an outgoing particle above the $\mathrm{EH}$, i.e, the outside particle cannot fall back on the black hole. Energy conservation requires that the particles inside, and outside the EH has the same value of $e$, and is created at equal distances from the $\mathrm{EH},\left(1-r_{\text {in }}=r_{\text {out }}-1\right)$. Global energy conservations force then the mass of the particle below the $\mathrm{EH}$ to be negative, and equal to minus the mass the particle above the $\mathrm{EH}$, i.e., the black hole looses energy as a consequence of pair production.

\section{Keywords}

Hawking Effect

\section{The Metric}

A transparent derivation of Painlevé-Gullstrand's coordinates (used in this paper) is the following: Consider a radially, free-falling observer that starts at rest from infinity. For this observer, the equations for $r$ as a function of the proper time $\tau$, and the equation for the Schwarzschild time, $t$, as a function of $r$, are respectively (cf. [1], Equations (9.38), (9.40)),

$$
r(\tau)=\left(\frac{3}{2}\right)^{\frac{2}{3}}(2 M)^{\frac{1}{3}}\left(\tau_{*}-\tau\right)^{\frac{2}{3}}
$$




$$
\begin{aligned}
t(r) & =t_{*}+2 M\left\{-\left(\frac{2}{3}\right)\left(\frac{r}{2 M}\right)^{\frac{3}{2}}-2\left(\frac{r}{2 M}\right)^{\frac{1}{2}}+\log \left|\frac{N}{D}\right|\right\} . \\
N & =\left(\frac{r}{2 M}\right)^{\frac{1}{2}}+1, D=\left(\frac{r}{2 M}\right)^{\frac{1}{2}}-1,
\end{aligned}
$$

where $\tau_{*}$ and $t_{*}$ are two constants. The equations are written in geometrized units. It follows from Equation (1a) that the second term in Equation (1b) is equal to $\tau-\tau_{*}$. This relation suggests the following transformation of coordinates,

$$
t=\tau+2 M\left\{-2\left(\frac{r}{2 M}\right)^{\frac{1}{2}}+\log \left|\frac{N}{D}\right|\right\}
$$

the constants in Equations (1a) and (1b) have been ignored because only $\mathrm{d} t$ is important. From Equation (2) we obtain,

$$
\mathrm{d} t=\mathrm{d} \tau-\mathrm{d} r\left(\frac{2 M}{r}\right)^{\frac{1}{2}}\left(1-\frac{2 M}{r}\right)^{-1} .
$$

which can also be derived directly from the equations,

$$
\frac{\mathrm{d} r}{\mathrm{~d} \tau}=-w, \frac{\mathrm{d} t}{\mathrm{~d} r}=-\frac{1}{w\left(1-w^{2}\right)}, w=\left(\frac{2 M}{r}\right)^{\frac{1}{2}},
$$

valid for a radial plunge with no kinetic energy at infinity. The new metric can be written,

$$
\mathrm{d} s^{2}=-\left(1-\frac{2 M}{r}\right) \mathrm{d} \tau^{2}+2\left(\frac{2 M}{r}\right)^{\frac{1}{2}} \mathrm{~d} r \mathrm{~d} \tau+\mathrm{d} r^{2}+r^{2}\left(\mathrm{~d} \theta^{2}+\sin ^{2} \theta \mathrm{d} \phi^{2}\right)
$$

The radially ingoing and outgoing light rays are found to be $(\mathrm{d} \tau / \mathrm{d} r$; it follows from the geodesic equations that $r$ is an affine parameter),

$$
\text { in }=-\frac{1}{(1+w)}, \text { out }=\frac{1}{(1-w)}, w=\left(\frac{2 M}{r}\right)^{\frac{1}{2}} .
$$

The condition $\mathrm{d} s^{2}=-\mathrm{d} \tau^{2}$ requires that,

$$
\left(\frac{2 M}{r}\right) \mathrm{d} \tau^{2}+2\left(\frac{2 M}{r}\right)^{\frac{1}{2}} \mathrm{~d} r \mathrm{~d} \tau+\mathrm{d} r^{2}=0
$$

the value of $\mathrm{d} r / \mathrm{d} \tau(=-w)$ obtained from Equation (6) agrees with Equation (1a).

\section{Particle Orbits}

\subsection{Expression for a Particle's Energy Per Unit Rest Mass}

We introduce the following change of notation: the time coordinate, $\tau$, in Equation (4) for the metric will be designated hereafter by $t$, whereas $\tau$ will be a particle's proper time. For radial motions the equations for $u^{r}=\mathrm{d} r / \mathrm{d} \tau$ and $u^{t}=\mathrm{d} t / \mathrm{d} \tau$ are,

$$
\boldsymbol{u} \cdot \boldsymbol{k} \boldsymbol{v}=\left(\frac{2 M}{r}-1\right) u^{t}+w u^{r}=-k,\left(u^{r}\right)^{2}=k^{2}+\frac{2 M}{r}-1, w=\left(\frac{2 M}{r}\right)^{\frac{1}{2}}
$$

where $\boldsymbol{u}=\left(u^{t}, u^{r}\right), \boldsymbol{k} \boldsymbol{v}$ is the Killing vector, namely $(1,0)$, and $k$ is a constant (the Killing constant). For metrics that are independent of $t$, an integral of the geodesic equation exists, that is given by the scalar product of $(1,0)$ 
with $\boldsymbol{u}$, which is Equation (7.1), namely the first equation in (7). Equation (7.2) follows then from the normalization condition for $\boldsymbol{u}$, i.e., $\boldsymbol{u}^{2}=-1$. In the derivation of this equation, terms in the product $k u^{r}$ appear, but they cancel. In Equation (7.1), $w$ is dimensionless and it is clear that one can assign to $k$ the dimension of a velocity (if dimensions are introduced the first term would be multiplied by $c$.) We assume that $k$ and $e$, the energy per unit rest mass, are related by, $k^{2}=2 e-1$, and find that Equation (7.2), i.e., the second equation in (7), can be written,

$$
e=1+\frac{\left(u^{r}\right)^{2}}{2}-\frac{M}{r}
$$

Little need is there to praise Equation (8)! Because $k$ is constant it follows from $k^{2}=2 e-1$, that $e$ is also a constant, and if $k=1$, also $e=1$.

Equation (8) is an expression for the conservation of the particle's energy as it moves along a timeindependent space-time geometry.

\subsection{Particle Orbits for Some Important Values of $k$}

In this section we study particle orbits only for values of $k=0,1$, because then (unlike other cases as, e.g., for $1>k>0$, in particular) solutions can easily be found. If $k=0$, and for radial motions, the equation for $\boldsymbol{u}$ is found to be,

$$
\boldsymbol{u}=\left\{w\left(w^{2}-1\right)^{-\frac{1}{2}},-\left(w^{2}-1\right)^{\frac{1}{2}}, 0,0\right\}, k=0
$$

We expect that for physically meaningful solutions the particle's proper time increases with the coordinate time, and have therefore discarded the solution with $\mathrm{d} t / \mathrm{d} \tau=-w\left(w^{2}-1\right)^{-\frac{1}{2}}$. Equation (9) is valid only below the event horizon and it follows from Equations (7.2) and (8) that the value of $-u^{r}$ given by Equation (9) is the minimum fall velocity, and furthermore that $e=\frac{1}{2}$. Therefore inside the event horizon, $e$ must be larger than $\frac{1}{2}$. Below the event horizon, the potential energy decreases, but this decrease is compensated by the increase in the minimum allowed value for $u^{r}$.

We proceed now to calculate the orbits for $|k|=1$. The value $k=1$, corresponds to the orbit of a free-falling observer that starts at rest from infinity. The value of $e$ for all the solutions with $|k|=1$ is unity. It will be shown that $k=-1$, defines the orbit of a particle, with the same energy per unit mass as the free falling observer, but confined below the event horizon. It is convenient to use an orthogonal system of coordinates associated with the falling observer. It can be readily verified that the vectors,

$$
\boldsymbol{e}_{0}=\{1,-w\}, \boldsymbol{e}_{1}=\{0,1\},
$$

form an orthonormal basis; the first one being timelike.

Notice that in this basis, the vector $\boldsymbol{u}$ in Equation (9), for $k=0$, and the Killing vector, $\boldsymbol{k} \boldsymbol{v}$, can be written,

$$
\boldsymbol{u}=\frac{(w, 1)}{\left(w^{2}-1\right)^{\frac{1}{2}}}, \boldsymbol{k} \boldsymbol{v}=\boldsymbol{e}_{0}+w \boldsymbol{e}_{1}=(1, w)
$$

where we have adopted the following convention: $\left\{\mathrm{v}_{0}, \mathrm{v}_{1}\right\}$, and $\left(\mathrm{v}_{0}, \mathrm{v}_{1}\right)$, denote vectors in the coordinate and orthonormal basis respectively. It is clear that in Equation (11), $\boldsymbol{u}^{2}=-1$, and that $\boldsymbol{u} \cdot \boldsymbol{k} \boldsymbol{v}=-k=0$.

Returning to the case $|k|=1$, the equations that need to be satisfied are,

$$
\boldsymbol{u} \cdot \boldsymbol{k} \boldsymbol{v}=-k,-\left(u^{t}\right)^{2}+\left(u^{r}\right)^{2}=-1,|k|=1 .
$$

The four solutions to Equation (12) are, 


$$
\begin{gathered}
\boldsymbol{u}=-k\left(\frac{\left(w^{2}+1\right)}{\left(w^{2}-1\right)}, \frac{2 w}{\left(w^{2}-1\right)}\right),|k|=1 \\
\boldsymbol{u}=(1,0), k=1 ; \boldsymbol{u}=(-1,0), k=-1
\end{gathered}
$$

We do not expect the particle's proper time to decrease while the coordinate time increases, we are therefore left with only three physically meaningful solutions. In the coordinate basis the components of these three solutions are,

$$
\begin{aligned}
& \boldsymbol{u}=\{1,-w\}, \boldsymbol{u}=\left\{-\frac{\left(w^{2}+1\right)}{\left(w^{2}-1\right)},+w\right\}, k=1 . \\
& \boldsymbol{u}=\left\{\frac{\left(w^{2}+1\right)}{\left(w^{2}-1\right)},-w\right\}, k=-1 .
\end{aligned}
$$

The solution with $k=1$, in Equation (13b), shows that $\boldsymbol{u}$ is the velocity of a particle that is stationary with respect to the falling observer, as expected from our choice of basic vectors in Equation (10). The same conclusion can be reached in the coordinate basis (cf. the first equation in (14a)), because it follows from Equation (1a), that $\mathrm{d} r / \mathrm{d} \tau=-w$.

Equation (14b) represents a sinking particle with $e=1$ and has no physical meaning above the event horizon because there, $\mathrm{d} t / \mathrm{d} \tau<0$. Conversely, the second equation in (14a) represents an outgoing particle with no physical meaning below the event horizon. Here, the minimum descent velocity is equal to $\left(w^{2}-1\right)^{\frac{1}{2}}$ from Equation (9), and $w$ in Equation (14b) must satisfy the inequality $w>\left(w^{2}-1\right)^{\frac{1}{2}}$, which is indeed the case.

\section{Particle Pair Creation and Hawking Effect}

The Killing vector, $\boldsymbol{k} \boldsymbol{v}=(1,0)$, is timelike above the event horizon (out), and spacelike below (in). In the quantum creation of a pair of particles, energy conservation requires that [1],

$$
-\boldsymbol{u}_{\text {in }} \cdot \boldsymbol{k} \boldsymbol{v}-\boldsymbol{u}_{\text {out }} \cdot \boldsymbol{k} \boldsymbol{v}=k_{\text {in }}+k_{\text {out }}=0 .
$$

Here, $\boldsymbol{u}_{\text {in }}, \boldsymbol{u}_{\text {out }}$ are the four-velocities of the created pair. Above the event horizon, $k_{\text {out }}$, must be positive because it is proportional to the particle's energy measured by an observer with velocity $\boldsymbol{k} \boldsymbol{v}$. Therefore $k_{\text {in }}=-\boldsymbol{u}_{\text {in }} \cdot \boldsymbol{k} \boldsymbol{v}$ must be negative and equal to $-k_{\text {out }}$. For values of $|k|=1$, it follows from Equation (14b), that the velocity of the created particle below the event horizon must be, $\boldsymbol{u}_{i n}=\left\{\left(w_{i n}^{2}+1\right) /\left(w_{i n}^{2}-1\right),-w_{i n}\right\}$. From the time dependence of the solutions in Equation (14a), it is apparent that $\boldsymbol{u}_{\text {out }}$ must then be taken equal to $\left\{-\left(w_{\text {out }}^{2}+1\right) /\left(w_{\text {out }}^{2}-1\right), w_{\text {out }}\right\}$, which is the outgoing solution for $k=1$. The particle above the event horizon cannot fall back into the black hole. Because $w_{\text {in }}$ and $w_{\text {out }}$ are both very approximately equal to one, it is straightforward to show that $u_{\text {in }}^{t}=u_{\text {out }}^{t}$ requires that Equation (16.1) below, be satisfied. Equation (16.2) follows from the relation, $e=\left(k^{2}+1\right) / 2$,

$$
1-r_{\text {in }}=r_{\text {out }}-1, e_{\text {in }}=e_{\text {out }},
$$

where $r_{\text {in }}$ and $r_{\text {out }}$ are the radial coordinates of the particles forming the pair, an intuitively attractive result.

In Equation (162), $e_{\text {in }}$ and $e_{\text {out }}$ are the energies of the respective particles divided by their mass. Because $e_{i n}=e_{\text {out }}$, global energy conservation requires that the mass of the particle below the $\mathrm{EH}$ be equal to minus the mass of the particle above the EH, i.e., the energy of the particle below the event horizon must be negative in agreement with Schutz's [2] and Carlip's [3] interpretation of the Hawking effect. The particle with negative mass survives for a finite amount of time before reaching the center of the black hole. But it is an unobservable particle and provides the formalism with the necessary degrees of freedom that allows for the correct interpretation of an observed particle at infinity escaping from the black hole. It would of course be of great interest to understand what happens for values of $k$ such that $0<k<1$ because then the outside particle cannot 
escape to infinity.

We calculate now the ratio $\mathrm{d} t / \mathrm{d} \tau$, where $t$ is the Schwarzschild time, and $\tau$ is the proper time of the particles at the event horizon. From Equation (3b) it follows that $\mathrm{d} t / \mathrm{d} t_{\text {(metric) }}=1 /\left(1-w^{2}\right)$, and then from Equation (14a) we obtain for an outside particle,

$$
\frac{\mathrm{d} t}{\mathrm{~d} \tau}=\frac{\left(1+w_{\text {out }}^{2}\right)}{\left(1-w_{\text {out }}^{2}\right)^{2}}=\frac{1}{2\left(1-w_{\text {out }}\right)^{2}} .
$$

In a theory of particle creation, the proper time should play the relevant role. Assume then that at the event horizon, $N$ particle-pairs are produced in a time $\mathrm{d} \tau$. Equation (17) shows that for the outside observer, $N$ particles will have been produced in the incomparably larger time, $\mathrm{d} t=\mathrm{d} \tau / 2\left(1-w_{\text {out }}\right)^{2}$, which suggests a weak observed productions of particles. However, the Hawking radiation from a black hole is also very weak. Field theory calculations show that black holes emits as though it were a black body with temperature,

$$
k_{b} T=\frac{\hbar c^{3}}{(8 \pi G M)},
$$

the notation being standard. The temperature, $T$, is truly the physical temperature of the black hole, not merely a quantity paying a role mathematically analogous to temperature in the laws of black hole mechanics (cf. [4], p.12). Mini black holes excepted, the emission is weak, and the creation of particles with finite mass, even neutrinos, must be weaker still. However, as the temperature increases, during the final stages of evaporation, the creation of particle-pairs with finite mass, could conceivably, become important. It is clear however that the answer to this issue lies far beyond the scope of this paper, and can only be obtained with the help of field theories capable of calculating particle creation in a curved space time (see, e.g., [5]).

\section{Acknowledgements}

Enlightening comments by Professor James Hartle on the first version of this article are acknowledged. I am grateful to Drs. Bertrand Chauvineau, Roger Clark, and Robert Low for helpful discussions.

\section{References}

[1] Hartle, J.B. (2003) Gravity. Addison Wesley, Boston, 291.

[2] Schutz, B. (2007) A First Course in General Relativity. Cambridge University Press, Cambridge.

[3] Carlip, S. (2004) Re: Hawking Radiation and Vacuum Fluctuation. http://sci.techarchive.net/sci.physcsresearch/2004-10

[4] Wald, R.M. (2001) The Thermodynamics of Black Holes. Living Reviews Relativity, 4, 6.

[5] Parker, L. (1969) Quantized Fields and Particle Creation in expanding Universes. Physical Review, $183,1057$. 